\title{
On the performance of switching methods in space division multiplexing based optical networks
}

\author{
Sridhar Iyer \\ Department of ECE, S.G. Balekundri Institute of Technology, Shivabasavanagar, Belagavi, KA, India
}

\begin{tabular}{|c|c|}
\hline Article Info & ABSTRACT \\
\hline Article history: & \multirow{9}{*}{$\begin{array}{l}\text { In the current work, for Space Division Multiplexing based Optical Networks } \\
\text { (SDM-b-OTNs), we investigate the performance of various switching methods } \\
\text { with a variation in traffic evolution over different time frame periods. Initially, } \\
\text { comparison of the existing methods viz., independent switching (InSw), } \\
\text { frequency switching (FqSw), and space switching (SpSw) demonstrates that } \\
\text { (i) over longer periods of time frame, FqSw provisions low network usage, and } \\
\text { (ii) SpSw offers low network usage for shorter periods of time frame; however, } \\
\text { as time frame increases to longer periods, SpSw starts to outperform InSw. } \\
\text { Next, we investigate a hybrid switching (HySw) method which begins by } \\
\text { implementing InSw and then shifts to the use of SpSw after the activation of } \\
\text { specific numbers of space channels. The simulation results demonstrate that } \\
\text { HySw provisions substantial savings on the costs incurred for switching, and } \\
\text { with lower space channel values it also offers a balance in the trade-off which } \\
\text { occurs between the costs associated for activating the space channels and that } \\
\text { incurred for switching. }\end{array}$} \\
\hline Received Feb 13, 2020 & \\
\hline Revised May 8, 2020 & \\
\hline Accepted May 19, 2020 & \\
\hline Keywords: & \\
\hline Network sharing & \\
\hline Resource allocation & \\
\hline Space division multiplexing & \\
\hline Switching & \\
\hline
\end{tabular}

This is an open access article under the CC BY-SA license.



\section{Corresponding Author:}

Sridhar Iyer,

Department of ECE,

S.G. Balekundri Institute of Technology,

Shivabasavanagar, Belagavi, KA, India.

Email: sridhariyer1983@gmail.com

\section{INTRODUCTION}

With an incremental increase in Internet traffic leading to an exponential growth of demands, it has been necessary to increase the corresponding optical network (OTN) capacity. However, with the OTN capacity increase, simultaneously, the industry also strives towards reducing associated networking costs [1]. For almost a decade, wavelength division multiplexed (WDM) OTNs have kept pace with the exponentially increasing capacity demands by deploying single mode fiber (SMF) and sharing numerous frequencies (or wavelengths) [2] enabled by wavelength selective switches (WSSs) that flexibly perform frequency switching (FqSw). However, once a deployed SMF is exhausted of capacity, an exponential increase in fibers amount will be required to meet requested capacity demand which requires novel network sharing strategies. The above scenario can be ameliorated by the adoption of space division multiplexing (SDM) technology which provides more capacity at reduced network costs simultaneously with better scalability and integration [3]. The SDM based OTNs (SDM-b-OTNs) provide freedom degrees in both, space and frequency wherein, every optical signal is associated to a frequency channel (FqChn) and a space channel ( $\mathrm{SpChn}$ ) which may be denoted as $F q(m \in\{1,2, \ldots . . M\})$ and $S p(n \in\{1,2, \ldots . . N\})$, respectively [4]. In SDM-b-OTNs, the deployed fiber capacity can be utilized efficiently as demand capacity of a source and destination pair may be approximately equal to the capacity which the fiber can provision; however, to further increase the efficiency, novel switching 
and sharing techniques will be required [5, 6]. The existing OTNs implement independent switching (InSw) which provides high flexibility but increases complexity of switching nodes [7, 8]. To reduce complexity, studies in $[9,10]$ have demonstrated implementation of (i) frequency switching (FqSw), which uses network nodes that switch all SpChns placed at certain frequency from any input to any output direction [11], and (ii) spatial switching ( $\mathrm{SpSw}$ ), which assigns spectral superchannels (or bandwidth of any fiber or any core) in a dynamic manner [11]. In the current work, considering the change in time frame and evolution of traffic over this time frame, we investigate the impact of the various switching methods on the performance of a SDM-bOTN which adopts multiple core fibers or SMF bundles as the fiber solution i.e., the type of transmission media in which the space superchannels are not coupled.

The authors in [9] have investigated deployment of reconfigurable add-drop multiplexers (ROADMs) for SDM-b-OTNs with flexible frequency grid and enabled by multiple core fibers; however, the authors have not addressed $\mathrm{SpSw}$ in the study. In [12,13], the authors have investigated different resource assignment strategies including FqSw; however, in both studies, the authors have not accounted for $\mathrm{SpSw}$. The authors in [14] have investigated various switching methods in regard to SDM-b-OTNs; however, $\mathrm{SpSw}$ is not accounted for within the study. In [15], the authors have addressed FqSw for investigating SDM-b-OTNs which demonstrate flexibility in both, space and frequency domains. The authors in [16] have not considered either $\mathrm{FqSw}$ or $\mathrm{SpSw}$ of space superchannels in their proposal of architecture on demand concept which is applicable to SDM-b-OTNs. In [17], the authors have considered $\mathrm{SpSw}$ for only certain $\mathrm{SpSns}$ amount to investigate SDM-b-OTN performance in data centers which utilizes various switching architectures. Lastly, the authors in $[4,8,10]$ have reviewed different SDM-b-OTN node architectures; however, unlike current study, authors have not accounted for change in time frame and evolution of traffic.

In the current work, initially, we compare performance of existing switching solutions viz., InSw, $\mathrm{FqSw}$, and $\mathrm{SpSw}$ by conducting extensive simulation experiments considering realistic network topologies and parameters. The results demonstrate that (i) over a longer time frame, $\mathrm{FqSw}$ provisions low network utilization, and (ii) $\mathrm{SpSw}$ offers low network utilization for shorter time frames; however, as the time frame increases to longer periods, $\mathrm{SpSw}$ starts to outperform $\mathrm{InSw}$. Next, for postponing the costs associated with activating a new SpChn, we propose a new hybrid switching ( $\mathrm{HySw}$ ) method which begins by implementing $\mathrm{InSw}$ and then shifts to use of FqSw after activation of specific numbers of SpChns. We compare the performance of existing switching solutions with $\mathrm{HySw}$ and results demonstrate that $\mathrm{HySw}$ postpones related SpChns activation costs simultaneously provisioning substantial savings on costs incurred for switching. Rest of the paper is structured as follows: In section 2, we detail the HySw method and also present the online (or dynamic) resource assignment algorithms applicable to every considered switching method. Section 3 presents the simulation setup and the various obtained simulation results and the corresponding discussions. Finally, section 4 concludes the study.

\section{HYBRID SWITCHING METHOD}

From the existing studies it is clear that on one hand, InSw requires lesser amount of SpChns and higher complexity of network switching, on the other hand, $\mathrm{SpSw}$ reduces the switching complexity by activating higher amounts of SpChns in the initial time frame periods. The proposed $\mathrm{HySw}$ method combines the best attributes of $\mathrm{InSw}$ and $\mathrm{FqSw}$. We propose $\mathrm{HySw}$ with a view to postpone the related $\mathrm{SpChns}$ (i.e. fibers or cores) activation costs simultaneously provisioning substantial savings on the costs incurred for the switching. The HySw method starts by implementing InSw at early stages of time frame and shifts to implementation of SpSw upon the activation of a certain ${ }^{N_{S p}}$ amount of SpChns. Hence, HySw splits SDM b-OTN into two layers which do not depend on one another and are activated at varied instant of time frame.

As an example, Figure 1 shows the evolution of implementation of $\mathrm{HySw}$ for $N_{S p}=2$. In such a scalable architecture, InSw is implemented for $N=1$ (see the $1^{\text {st }}$ column), and for $N=2$ (see the $2^{\text {nd }}$ column). However, if new (see the $3^{\text {rd }}$ and $4^{\text {th }}$ coloumn) SpCns are activated then, $\mathrm{SpSw}$ is implemented ensuring that the nodes which were deployed to implement $\mathrm{InSw}$ initially remain active and operational. Overall, the major idea of implementing $\mathrm{HySw}$ is that initially, with InSw, since lesser SpChns will be activated, finer value of granularity can be used to switch the signals; at later stages, when the active SpChns amount increases, with $\mathrm{SpSw}$, frequency clashes can be prevented and in turn the nodes complexity can be minimized. Hence, there occurs a postponement in SpChns being activated which will result in reduction of the associated costs. Although, $\mathrm{SpSw}$ will need $\mathrm{SpChns}$ to be activated untimely, a combination of $\mathrm{InSw}$ and $\mathrm{SpSw}$, as in $\mathrm{HySw}$, will alleviate the aforementioned scenario. Also, for SpChns to be activated, assuming that network operator has fiber solution available at his end, optical amplifiers and optical switching matrices will be required to be deployed in the entire network which will incur increased installation costs at early time frame. However, as the major benefits, (i) in comparison to the use of WSSs by InSw, SpSw uses optical switching matrices which 
are much cheaper and also minimize costs associated with regeneration of the optical signals, and (ii) use of the SpChns can be avoided for longer time frames by implementing SpSw which will postpone deployment of new fiber solutions.

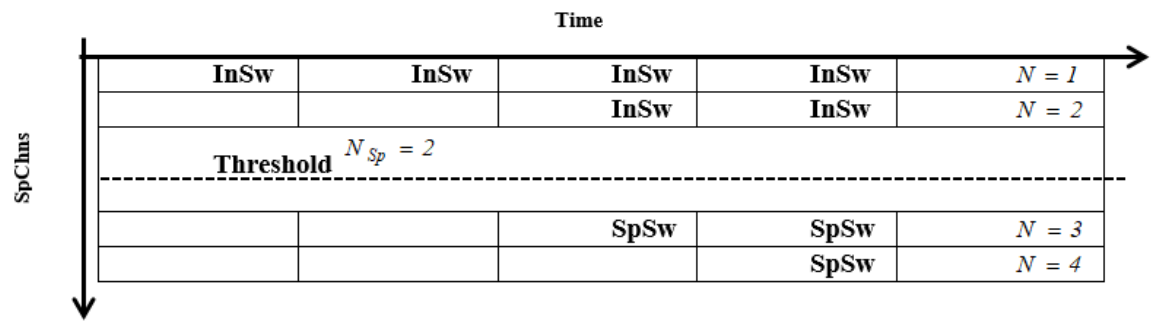

Figure 1. Working of HySw method with evolution of time frame considering threshold value of $N_{S p}=2$

\subsection{Online resource assignment algorithms}

In In this sub-section, we detail the online resource assignment algorithms which are applicable to the considered switching methods. When InSw is to be implemented then initially, $k$-shortest path $(k$-SP) algorithm is used to route the signals following which first fit wavelength assignment method is used to allocate the frequencies (or wavelengths). A demand is accepted in case when the desired capacity is available; however, activation of a new SpChn over the entire SDM-b-OTN links is conducted when the desired capacity is not found and the algorithm is then restarted for acceptance of the demand which is incoming. It must be noted that a demand which is incoming can be accepted by utilizing a space superchannel which exists and has the same source and destination pair by virtue of the activation of a new SpChn which results in creation of extra capacity for all space superchannel that exist.

When $\mathrm{FqSw}$ is to be implemented then initially, to serve the demand, the algorithm starts by checking whether there already exists a space superchannel which has been assigned with the available desired capacity. If such a space superchannel exists then, demand is provisioned with existing available capacity; else, algorithm uses $k$-SP to determine a space superchannel which is available. Next, demand is served if the algorithm is able to assign the space superchannel which is available; else, activation of a new SpChn over the entire SDM-bOTN links is conducted and the algorithm is then restarted for the acceptance of the demand which is incoming. When $\mathrm{SpSw}$ is to be implemented then initially, algorithm determines the existence of an assigned spectral superchannel which has the available desired capacity, and if it finds such a spectral superchannel, demand is served using the found capacity. However, if such a spectral superchannel is not found then algorithm uses $k$ SP to determine a spectral superchannel with available desired capacity. Upon the successful searching, algorithm serves demand by assigning the determined spectral superchannel; else, activation of a new SpChn over the entire SDM-b-OTN links is conducted and the algorithm is then restarted for the acceptance of the demand which is incoming. Finally, when HySw is to be implemented then, the algorithm executes InSw till the activation of $N_{S p}$ SpChsn following which the algorithm executes $\mathrm{SpSw}$ for the remaining time frame.

\section{SIMULATION RESULTS AND DISCUSSIONS}

To compare performance of considered switching methods, simulation experiments are conducted such that the connection demands which are accepted within the network are never torn down. Considering the fact that activation of a new SpChn over all links results in provisioning extra capacity to serve a new demand, simulations are conducted in a manner which leads to no blocking of the demands. Also, between all the source and destination pairs, uniform distribution is followed, and space and frequency conversion is not permitted in the simulations. Further, to determine the candidate paths for every source-destination nodes pairs, we use $k$-SP with a fixed value of $k=3$ which has been shown to demonstrate the best performance in the SDM-b-OTNs [18]. The number of frequency slots considered is 96 with each occupying $50 \mathrm{GHz}$ of spectrum which amount to a total of $4.8 \mathrm{THz}$ bandwidth [19]. Further, the considered demands have a fixed capacity of $100 \mathrm{Gbps}$ which are assigned over a fixed $50 \mathrm{GHz}$ frequency slot. Each run of the simulation is determined as the mean of 50 runs. For performance evaluations, we consider two realistic network topologies: small distance DT, and larger distance GEANT [20-21]. In regard to capacity, following [22] which predict the compound annual growth rates (CAGRs) ranging between $20 \%$ - 50\% depending on the geographic regions, starting value in the $1^{\text {st }}$ year is set to $5 \mathrm{Tbps}$ which is then increased every year following CAGR of $20 \%$ or 
40\%. As the performance metrics which undergo a change every year, we use the amount of (i) aggregate active SpChns (AgAcSpChns), and (ii) network usage (NetUsg) (in \%) which is evaluated as NetUsg $=\sum_{j=1}^{d} H_{j} \cdot F S_{j} / F S s p \cdot A c S p \cdot E d$

$[1,4]$ where, in numerator, generated demands amount is denoted by $d$, $H_{j}$ denotes hops amount which a demand $d$ traverses, and $F S_{j}$ denotes frequency slots amount which a demand ${ }^{d}$ utilizes. Further, in denominator, ${ }^{F S s p}$ denotes frequency slots amount which every SpCh supports, $A c S p$ denotes amount of SpChns which are active, and $E d$ denotes amount of network graph's edges.

\subsection{Performance comparison of existing switching methods}

In this sub-section, we present performance comparison results of the existing switching methods. The results are presented in Figure 2 considering the CAGR values of $20 \%$ and $40 \%$. From the results it can be seen that trends of results is the same for both the considered topologies. Specifically, for both topologies, only two SpChs are needed by $\mathrm{InSw}$ and $\mathrm{FqSw}$ for a time frame period of 7 and 5 years corresponding to a CAGR value of $20 \%$ and $40 \%$, respectively. Hence, it can be inferred that at lower values of network loads both, InSw and FqSw incur lower associated investment costs. Further, in regard to utilization of the network, it can be observed that both, InSw and $\mathrm{FqSw}$ achieve highest performance of approximately $40 \%$ in the initial periods of time frame. Henceforth, for $\mathrm{FqSw}$, NetUsg value minimizes and remains below $25 \%$. It must be noted that highest NetUsg values of InSw and $\mathrm{FqSw}$ corresponds to the fact that the very first $\mathrm{SpCh}$ has exhausted the capacity and a second $\mathrm{SpCh}$ activation is required which minimizes usage of network. Further, after the aforementioned juncture is reached, AgAcSpChns is observed to incur an exponential increase whereas, NetUsg values continue to have lower values. Hence, it can be inferred that FqSw is not a favourable switching solution for longer periods of time frame even though efficient frequency allotment methods maybe used which however will only slightly increase spectral-efficiency. It can also be seen that InSw is able to provision higher network usage values of approximately $45 \%$ for longer time frame periods.
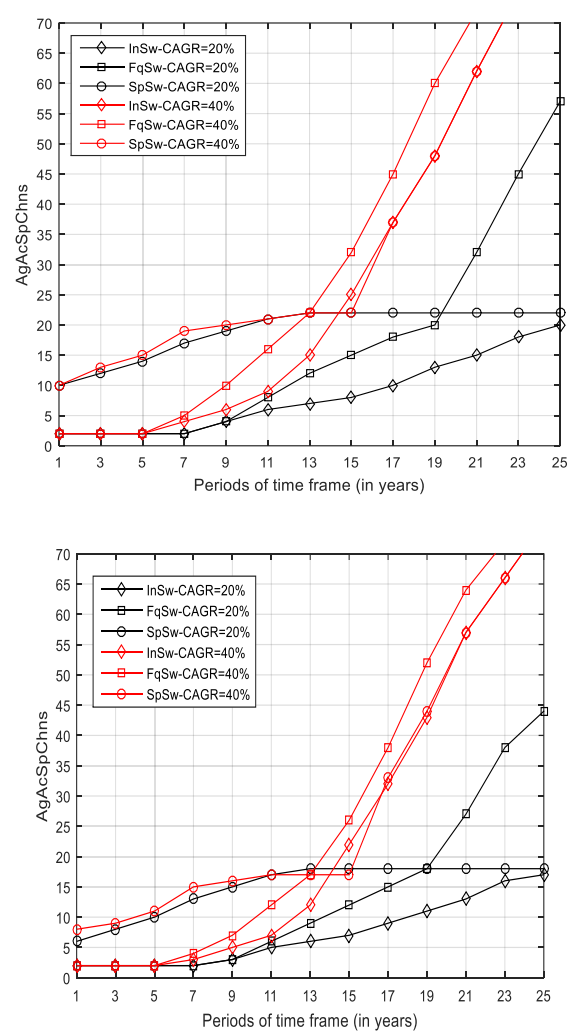

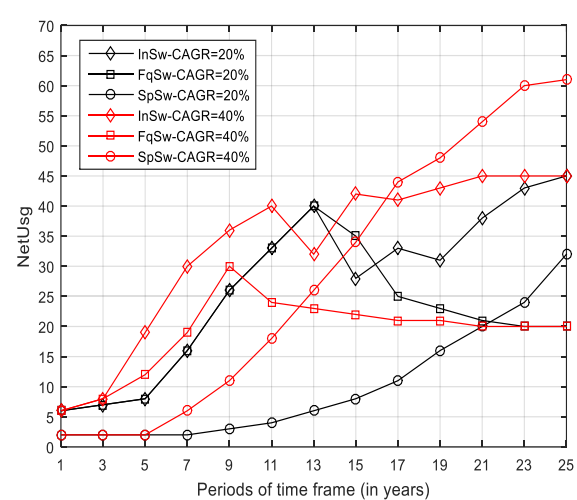

(a)



(b)

Figure 2. AgAcSpChns (left) and NetUsg (right) results for CAGR values of $20 \%$ and $40 \%$ considering the network topologies (a) DT, and (b) GEANT 
The SpSw method is observed to activate higher SpChns amount in the initial time frame periods since it needs to connect all the source and destination pairs in the SDM-b-OTN through individual SMF, and hence, usage of network has a lower values until such a connection is completed. As the time frame periods increase, $\mathrm{SpSw}$ is seen to outperform $\mathrm{InSw}$ even though $\mathrm{SpSw}$ has much lower flexibility of switching. The aforementioned occurs since $\mathrm{SpSw}$ does not incur any substantial capacity losses owing to frequency clashing, and the same can be observed when CAGR value is $40 \%$ under which, $\mathrm{SpSw}$ has an approximate NetUsg value of $60 \%$ and $75 \%$ for the DT and the GEANT network topology, respectively.

\subsection{Performance comparison of existing switching methods with hybrid switching method}

In this sub-section, we present the performance comparison results of the existing switching methods with the proposed HySw method. For the HySw method, InSw is implemented until certain $N_{S p}$ amounts of SpChns have been activated following which, the $\mathrm{SpSw}$ method is implemented. In the simulations, we vary the value of $N_{S p}$ as $N_{S p} \in\{2,4,6,8,10\}$. The simulation experiment results are presented in Figures 3 and 4 for the DT and the GEANT network topologies considering the CAGR values of $20 \%$ and $40 \%$, respectively.

Specifically, from Figure 3 it can be observed that the HySw method for a lower value of $N_{S p}=2$ leads to the postponement of larger amount of SpChns by approximately 9 to 10 years and simultaneously ensures that the complexity of the network nodes is low. Further, for longer periods of time frame, in comparison to the $\mathrm{SpSw}$ method, this low $N_{S p}=2$ value is also seen to minimize the active SpChns amount since it provisions a layered network which comprises of a single dimension and granularity of the frequency. Also, in the later periods of the time frame owing to the low values of NetUsg, it can be observed that the use of the HySw method results in an increased active dimensions amount. The aforementioned is a result of the fact that when the $\mathrm{SpSw}$ method is implemented at the later periods of the time frame, it leads to a change-over stage wherein, the HySw method results in the activation of more SpChns amount in comparison to the $\mathrm{SpSw}$ method.
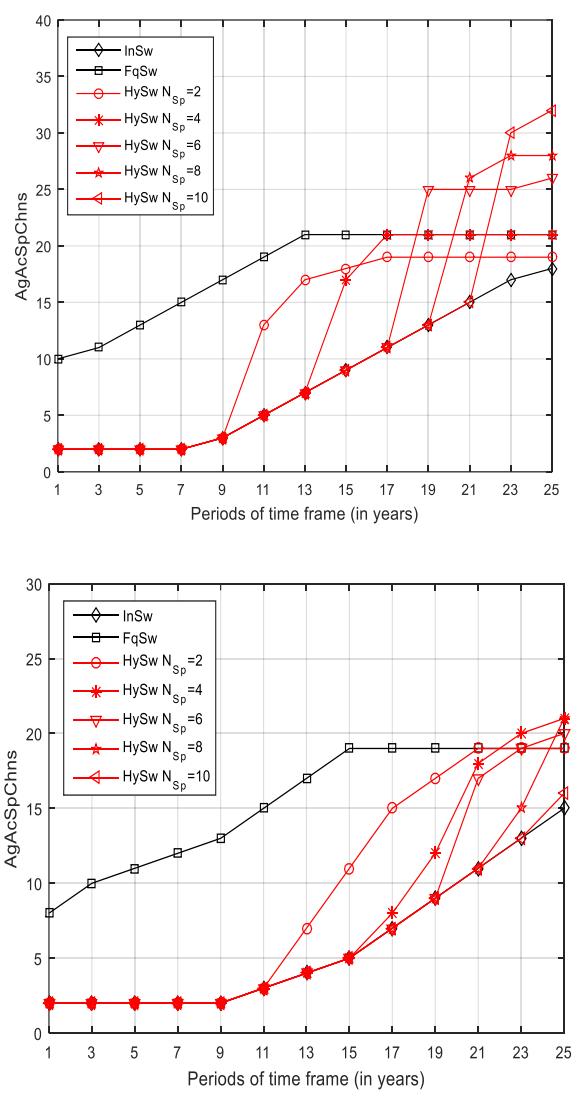

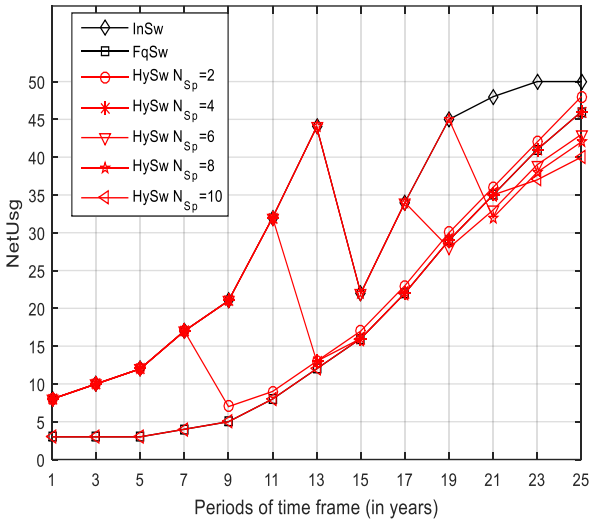

(a)

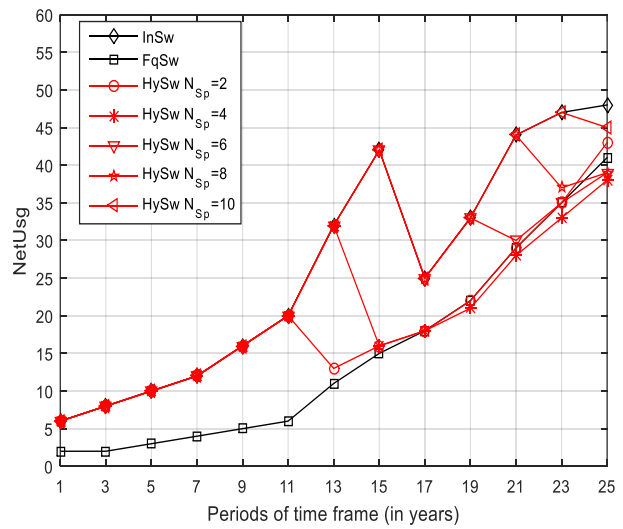

(b)

Figure 3. AgAcSpChns (left) and NetUsg (right) results for a CAGR value of $20 \%$ considering the network topologies (a) DT, and (b) GEANT 

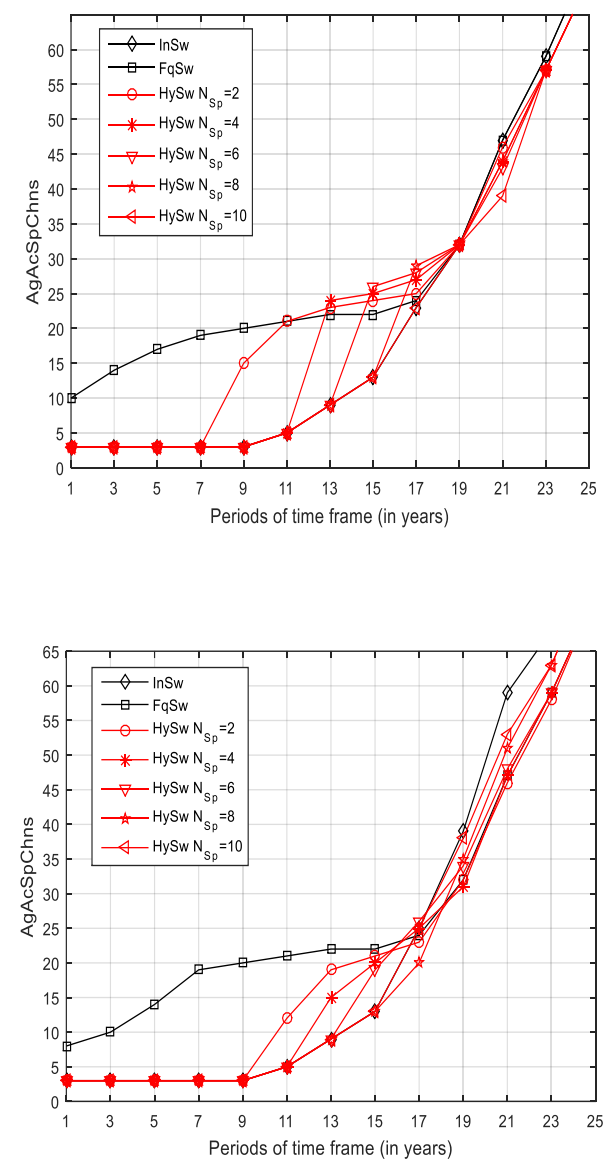

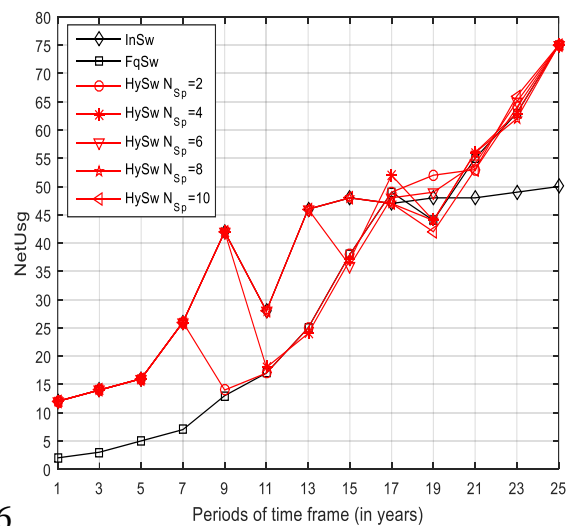

(a)

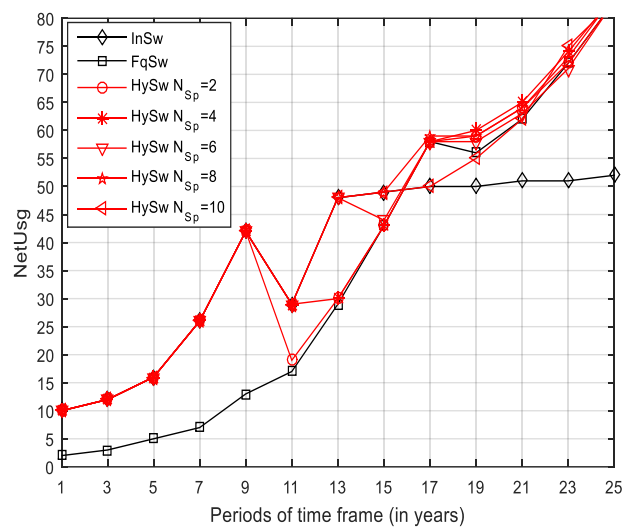

(b)

Figure 4. AgAcSpChns (left) and NetUsg (right) results for a CAGR value of $40 \%$ considering the network topologies (a) DT, and (b) GEANT

The aforementioned high values of NetUsg provisioned by $\mathrm{SpSw}$ for heavily loaded traffic demands can be seen more apparently in Figure 4. Also, for later periods of time frame, irrespective of $N_{S p}$ value, it is observed that HySw merges to a single NetUsg value which is much higher than that reached by InSw. Hence, from all the obtained results it can be inferred that there is a trade-off between the costs associated for activating the SpChns and for switching. Further, the use of the HySw method with lower ${ }^{N_{S p}}$ values is able to offer a balance between the aforementioned costs.

\section{CONCLUSION}

In the current work, we investigated performance of the switching solutions which are applicable to SDM-b-OTNs. The results revealed that (i) compared to other switching solutions, FqSw demonstrates lower network utilization over a longer period of time frame, and (ii) $\mathrm{SpSw}$ outperforms InSw for longer time frame periods since it is able to avoid both, fragmentation of spectrum and losses of capacity owing to frequency clashing. Further, results also demonstrated that for shorter periods of time frames, SpSw offers low network utilization as it activates large amounts of SpChns. Considering the aforementioned, we proposed the HySw strategy which reduces costs associated with activating a new SpChn as it begins by implementing InSw and then shifts to the use of FqSw after activation of specific numbers of SpChns. The results demonstrated that HySw maintains the spectral-efficiency over a longer time frame and provisions substantial savings on the incurred switching costs. As an important observation, it is found that in the later periods of time frame, use of HySw results in an increased active dimensions amount since, use of $\mathrm{SpSw}$ at later periods of time frame leads to a change-over stage wherein, HySw results in the activation of more SpChns amount in comparison to SpSw. It is also observed that the use of HySw with lower $N_{S p}$ values is able to offer a balance in trade-off which occurs between costs associated for activating the SpChns and for switching. 


\section{REFERENCES}

[1] E. Agrell, et al., "N. Gisin. Roadmap of optical communications", Journal of Optics, IOP Press, vol. 18, no. 1, pp. $1-40,2016$.

[2] S.P. Singh, et al., "Study on Mitigation of Transmission Impairments and Issues and Challenges with PLIA-RWA in Optical WDM Networks", Journal of Optical Communication, De Gruyter, vol. 33, no. 2, pp.83-101, 2012.

[3] T.J. Xia, et al., "Introduction of spectrally and spatially flexible optical networks", IEEE Communications Magazine, vol. 53, no. 2, pp. 24-33, 2015.

[4] D. Klonidis, et al., "Spectrally and Spatially Flexible Optical Network Planning and Operations", IEEE Communications Magazine, vol. 53, no. 2, pp. 69-78, 2015.

[5] P.J. Winzer "Spatial multiplexing in fiber optics: The 10× scaling of metro/core capacities", Bell Labs Technical Journal, vol. 19, pp. 22-30, 2014.

[6] G. M. Saridis, et al., "Survey and evaluation of space division multiplexing: From technologies to optical networks", IEEE Communications Surveys and Tutorials, vol. 17, no. 4, pp. 2136-2156, 2015.

[7] P.J. Winzer, et al., "From scaling disparities to integrated parallelism: A decathlon for a decade", IEEE Journal of Lightwave and Technology, pp. 35, no. 5, pp. 1099-1115, 2017.

[8] D.M. Marom, et al., "Switching solutions for WDMSDM optical networks", IEEE Communications Magazine, vol. 53, no. 2, pp. 60-68, 2015.

[9] F.J. Moreno-Muro, et al., "Evaluation of Core-Continuity-Constrained ROADMs for Flex-Grid/MCF Optical Networks", IEEE/OSA Journal of Optical Communications and Networking, vol. 9, no. 11, pp. 1041-1050, 2017.

[10] D.M. Marom, et al., "Survey of Photonic Switching Architectures and Technologies in Support of Spatially and Spectrally Flexible Optical Networking", IEEE/OSA Journal of Optical Communications and Networking, vol. 9, no. 9, pp. 1-26, 2017.

[11] N. K. Fontaine, et al., "Heterogeneous space-division multiplexing and joint wavelength switching demonstration", in IEEE/OSA Optical Fiber Communications Conf. (OFC), pp. 1-3, 2015.

[12] P. S. Khodashenas, et al., "Comparison of Spectral and Spatial Superchannel Allocation Schemes for SDM Networks", IEEE/OSA Journal of Lightwave Technology, vol. 34, no. 11, pp. 2710-2716, 2017.

[13] D. Siracusa, et al., "Resource allocation policies in SDM optical networks", in IEEE International Conference Optical Network Design and Modeling (ONDM), pp. 168-173, 2015.

[14] B. Shariati, et al., "Impact of spatial and spectral granularity on the performance of SDM networks based on spatial superchannel switching", IEEE/OSA Journal of Lightwave Technology, vol. 35, no. 13, pp. 2559-2568, 2017.

[15] C. Rottondi, et al., "Routing, modulation format, baud rate and spectrum allocation in optical metro rings with flexible grid and few-mode transmission", IEEE Journal of Lightwave Technology, vol. 35, no. 1, pp. 61-70, 2017.

[16] A. Muhammad, et al., "Resource allocation for space-division multiplexing: optical white box versus optical black box networking”, IEEE Journal of Lightwave Technology, vol. 33, no. 23, pp. 4928-4941, 2015.

[17] M. Fiorani, et al., "Spatial division multiplexing for high capacity optical interconnects in modular data centers", IEEE/OSA Journal of Optical Communications and Networking, vol. 9, no. 2, pp. A143-A153, 2017.

[18] J. Perelló, et al., "Flex-grid/SDM backbone network design with intercore XT-limited transmission reach", IEEE/OSA Journal of Optical Communications and Networking, vol. 8, no. 8, pp. 540-552, 2016.

[19] S. Iyer, et al., "Spectral and Power-Efficiency Investigation in Single and Multi-Line- Rate Optical Wavelength Division Multiplexed (WDM) Networks", Photonics Network Communications, Springer, vol. 33, no. 1, pp. 39-51, 2017.

[20] S. Iyer, "On Routing, Modulation Format, Space and Spectrum Allocation with Protection in Space Divison Multiplexing based Elastic Optical Networks", Journal of Information and Telecommunication, Taylor \& Francis, vol. 4, no. 1, pp. 105-117, 2020.

[21] S. Iyer, "An Online Routing Algorithm for Space Division Multiplexing Based Elastic Optical Networks", International Journal of Communication Networks and Distributed Systems, Inderscience, vol. 24, no. 2, pp. 167185,2020

[22] Cisco Visual Networking Index: Forecast and Methodology, 2016-2021, White paper, 2017. https://www .cisco.com/c/en/us/solutions/collateral/service-provider/visualnetworking-index-vni/complete-white-paper-c11481360.pdf 